\title{
The molecular mechanism linking muscle fat accumulation to insulin resistance
}

\author{
Matthew W. Hulver and G. Lynis Dohm* \\ Department of Physiology, Brody School of Medicine, East Carolina University, Greenville, NC 27858, USA
}

\begin{abstract}
Skeletal muscle insulin resistance is a co-morbidity of obesity and a risk factor for the development of type 2 diabetes mellitus. Insulin resistance is associated with the accumulation of intramyocellular lipids. Intramyocellular triacylglycerols do not appear to be the cause of insulin resistance but are more likely to be a marker of other lipid intermediates such as fatty acyl-CoA, ceramides or diacylglycerols. Fatty acyl-CoA, ceramides and diacylglycerols are known to directly alter various aspects of the insulin signalling cascade. Insulin signalling is inhibited by the phosphorylation of serine and threonine residues at the levels of the insulin receptor and insulin receptor substrate 1 . Protein kinase $\mathrm{C}$ is responsible for the phosphorylation of the serine and threonine residues. Fatty acyl-CoA and diacylglycerols are known to activate protein kinase $\mathrm{C}$. The cause of the intramyocellular accumulation of fatty acyl-CoA and diacylglycerols is unclear at this time. Reduced fatty acid oxidation does not appear to be responsible, as fatty acyl-CoA accumulates in skeletal muscle with a normal fatty acid oxidative capacity. Other potential mechanisms include oversupply of lipids to muscle and/or up regulated fatty acid transport.
\end{abstract}

Insulin resistance: Intramyocellular lipids: Fatty acyl-CoA: Diacylglycerols

Skeletal muscle insulin resistance is characterized by blunted insulin-stimulated glucose uptake and metabolism. Insulin resistance is a co-morbidity of overweight and obesity and is a risk factor for the development of type 2 diabetes and CVD. It has been well documented that insulin-stimulated glucose transport is markedly blunted in skeletal muscle of obese individuals and in patients with type 2 diabetes (Dohm et al. 1988). Since skeletal muscle is the primary site of glucose disposal in the human body, the inability of this tissue to take up glucose in response to insulin most probably explains the reduced in vivo disposal of glucose observed in insulin-resistant individuals. It is well established in the literature that the accumulation of intramyocellular lipids is associated with insulin resistance (McGarry, 2002). The purpose of the present paper is to show the link between intramyocellular lipid accumulation and skeletal muscle insulin resistance. Evidence will be provided that human skeletal muscle insulin resistance is a result of defects in the insulin signalling cascade that are mediated by protein kinase $\mathrm{C}$ (PKC) and that intramuscular lipids activate PKC. In addition, a hypothesis will be provided as to why lipids accumulate in insulin-resistant skeletal muscle.

\section{Relationship of skeletal muscle insulin resistance and intramyocellular lipid accumulation}

It is well established that intramyocellular triacylglycerol accumulation is associated with skeletal muscle insulin resistance (McGarry, 2002). However, it is unlikely that triacylglycerols are responsible for reduced skeletal muscle insulin action, but it is more likely that they are an inert marker of other lipid intermediates known to suppress insulin sensitivity. An increase in the intramyocellular concentration of lipid intermediates such as fatty acyl-CoA, ceramides and diacylglycerols (DAG) not only correlate with insulin resistance, but also directly and indirectly alter insulin signalling (Schmitz-Peiffer, 2002). Considerable evidence linking increased skeletal muscle lipid content to insulin resistance has been derived from animal studies employing acute and chronic high-fat diets (Cooney et al. 2002). Fatty acid-induced insulin resistance appears to occur in concert with increased fatty acyl-CoA (Cooney et al. 2002). Moreover, insulin sensitivity is restored by treatments that reduce intramyocellular lipid accumulation (i.e. low-fat feeding, fasting and exercise; Oakes et al. 1997). Similar findings have been observed in human 
subjects. Bachmann et al. (2001) have reported marked increases in intramyocellular lipid content and reductions in insulin sensitivity following intravenous lipid infusion. Ellis et al. (2000) have demonstrated a negative correlation between insulin-mediated glucose disposal and fatty acyl-CoA content in skeletal muscle from a group of older men. Blunted insulin-mediated glucose disposal has been observed in skeletal muscle from moderately- and morbidly-obese patients (Fig. 1(a)), which is accompanied by elevated levels of intramyocellular fatty acyl-CoA (Fig. 1(b)). In contrast, intramyocellular triacylglycerols are only elevated in the morbidly-obese skeletal tissue (Fig. 1(c)). Thus, fatty acyl-CoA, or a derived lipid, are related to, and possibly responsible for, muscle insulin resistance.

\section{Early steps in insulin signalling are decreased in insulin-resistant muscle}

The first step in determining what causes skeletal muscle insulin resistance is to discern whether the defects exist in GLUT4 translocation or insulin signalling. Exercise and muscle contraction are known to stimulate skeletal muscle glucose transport, and contraction combined with insulin produce additive effects on glucose transport. These findings suggest that contraction and insulin stimulate glucose transport via two distinctly different mechanisms. In a series of experiments designed to test whether glucose transport can be stimulated in insulin-resistant muscle if a signal other than insulin is presented, it has been found that muscle contraction robustly stimulates glucose transport in muscle from obese Zucker rats (Dolan et al. 1993). In human muscle preparations, using hypoxia as a stimulus, it has been shown that glucose transport can be stimulated in obese insulin-resistant tissue (Azevedo et al. 1995). Stimulation of transport by hypoxia and muscle contraction occur by the same signalling pathway. These findings seem to confirm, therefore, that the glucose transport system (i.e. translocation and activation of glucose transporters) is intact in insulin-resistant muscle.

In a series of experiments that measured the early steps of the insulin-signalling pathway in normal and insulinresistant muscle it has been found that autophosphorylation of the insulin receptor, phosphorylation of insulin receptor substrate 1 and activation of phosphotidylinositol 3-kinase are all depressed in incubated human muscle from obese insulin-resistant patients (Goodyear et al. 1995). It has also been shown that insulin-receptor tyrosine kinase activity is reduced in muscle from obese patients and patients with type 2 diabetes (Caro et al. 1987; Zhou et al. 1999; Itani et al. 2000). These defects in insulin signalling are believed to be a result of phosphorylation of the insulin receptor on serine and threonine residues. To further examine this possibility, the effect of removing phosphates from the insulin receptor has been investigated by measuring tyrosine kinase activity in isolated insulin receptors from rat and human skeletal muscle with and without alkaline phosphatases treatment. In both rats and human subjects alkaline phosphatases increase insulin-stimulated tyrosine kinase activity. Interestingly, the effect of removing the phosphate is much greater in insulin receptors from obese insulin-resistant skeletal muscle as compared with lean insulin-sensitive muscle (Zhou et al. 1999; Itani et al. 2000). This finding suggests that insulin receptors from insulin-resistant muscles are more highly phosphorylated on serine and threonine residues than those of insulinsensitive muscles.

\section{Activation of protein kinase $\mathbf{C}$ causes insulin resistance}

PKC can directly phosphorylate and inactivate the insulin receptor. Likewise, overexpression of PKC isoforms in cultured cells causes phosphorylation of the insulin receptor and insulin resistance (Bossenmaier et al. 1997). These findings have led to the hypothesis in the scientific literature that PKC causes insulin resistance in skeletal muscle. To determine whether one of the PKC isoforms may be increased in insulin resistant muscle to cause phosphorylation of the insulin receptor, the protein content of eight PKC isoforms in the particulate fractions of muscles from lean and obese patients has been measured. The only PKC isoform that is increased in the insulinresistant muscle (obese) is PKC $\beta$. Basal PKC $\beta$ is higher in the in vitro incubated muscle from obese individuals and insulin increases PKC $\beta$ in the particulate fraction in muscle from obese patients but not in muscle from lean patients (Itani et al. 2000).

To demonstrate a cause and effect relationship between PKC activity and insulin action, human muscle strips have been incubated in the presence and absence of PKC activators and inhibitors. In insulin-resistant muscle the PKC inhibitor GF-109203X enhances insulin stimulation of glucose transport. In insulin-sensitive muscle incubation with the PKC activator 12-deoxyphorbol 13-phenylacetate causes insulin-stimulated glucose transport to be depressed (Cortright et al. 2000). These data suggest that a PKC activator can cause insulin resistance and that a PKC inhibitor can reverse insulin resistance. Furthermore, using mouse PKC knock-out and overexpression models, it has been possible to demonstrate increases and decreases in insulin sensitivity respectively in the gastrocnemius muscle (S Itani and GL Dohm, unpublished results).

Other research groups have made observations that also implicate PKC in the development of lipid-induced insulin resistance. Griffin et al. (1999), using a rat model, induced insulin resistance with a $5 \mathrm{~h}$ lipid-heparin infusion and observed a $50 \%$ reduction in insulin-stimulated insulin receptor substrate 1-associated phosphotidylinositol 3kinase activity, blunted insulin receptor substrate 1 autophosphorylation and a 4-fold increase in PKC activation. In human subjects Itani et al. (2002), also employing a lipid-heparin infusion but following $6 \mathrm{~h}$ of infusion, found that insulin-stimulated glucose disposal is reduced by $43 \%$, and skeletal muscle DAG mass and PKC activity is increased 4-fold. These findings not only imply that PKC activation causes skeletal muscle insulin resistance but also demonstrate an association between DAG and PKC activation.

\section{Intramyocellular lipids activate protein kinase $\mathbf{C}$}

Various intramyocellular lipid intermediates such as fatty acyl-CoA, ceramides and DAG inhibit specific steps in 

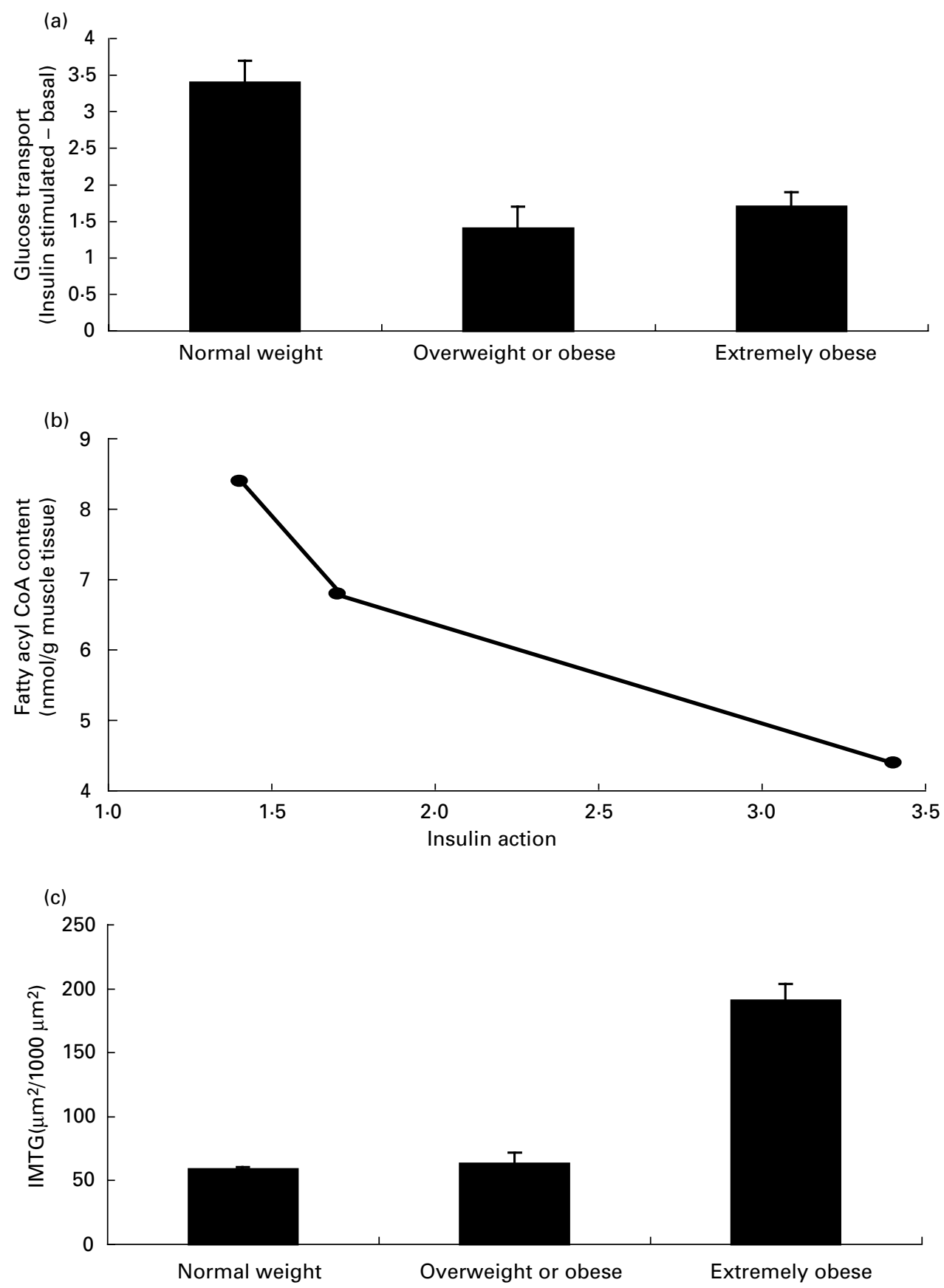

Fig. 1. Insulin action and lipid content in skeletal muscle from moderately- and morbidly-obese patients. (a) Glucose transport was measured in rectus abdominus strips with and without insulin, as previously reported (Dohm et al. 1988). Insulin action is expressed as insulin-stimulated minus basal glucose transport. (b) Association between insulin action and intramyocellular fatty acyl-CoA content. (c) Intramyocellular triacylglycerol (IMTG) content from vastus lateralis muscle samples. (a, c), Values are means with their standard errors represented by vertical bars. (Redrawn from Hulver et al. 2003.)

the insulin-signalling cascade (Schmitz-Peiffer, 2002). Ceramides activate a protein phosphatase that dephosphorylates akt/protein kinase B resulting in inhibition of GLUT4 translocation and glycogen synthesis (Long \& Pekala, 1996; Chavez et al. 2003). Fatty acyl-CoA and DAG have been implicated in the activation of PKC in various tissues (Shulman, 2000; Cooney et al. 2002;
Schmitz-Peiffer, 2002). Fatty acyl-CoA have been shown to directly activate PKC in brain tissue (Shoyab, 1985; Bronfman et al. 1988) but these observations have yet to be observed in skeletal muscle. However, in models of high fat exposure intramyocellular fatty acyl-CoA are elevated in concert with increased PKC activation (Itani et al. 2002). Moreover, fatty acyl-CoA are also implicated 
(a)

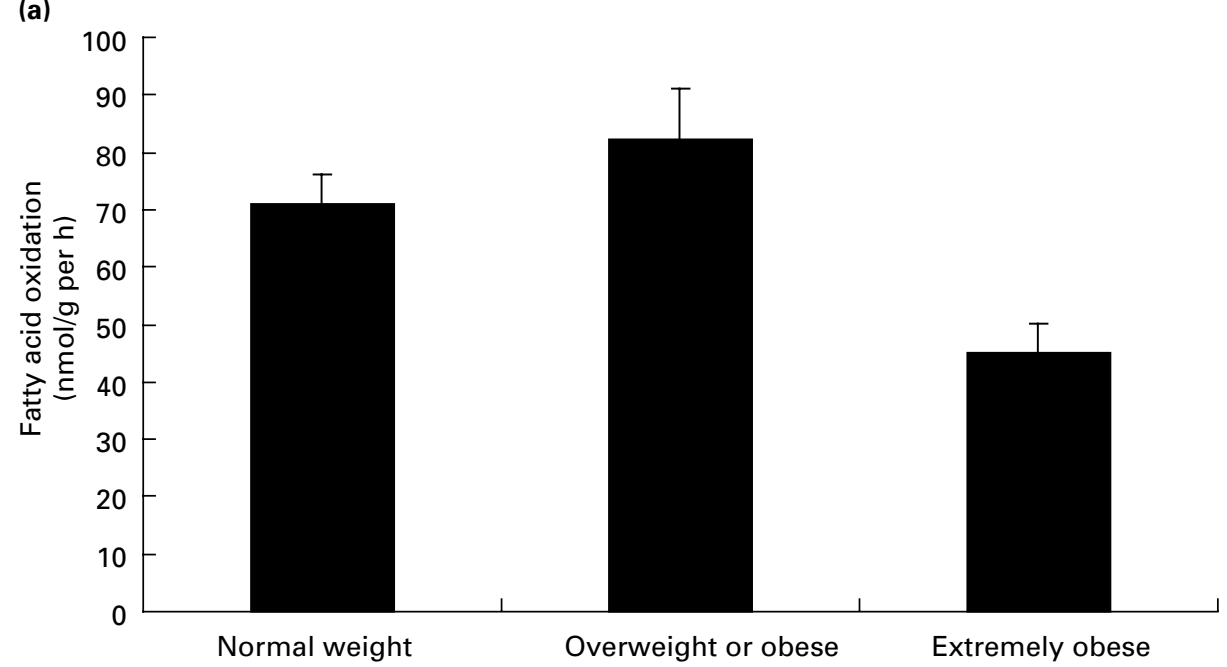

(b)

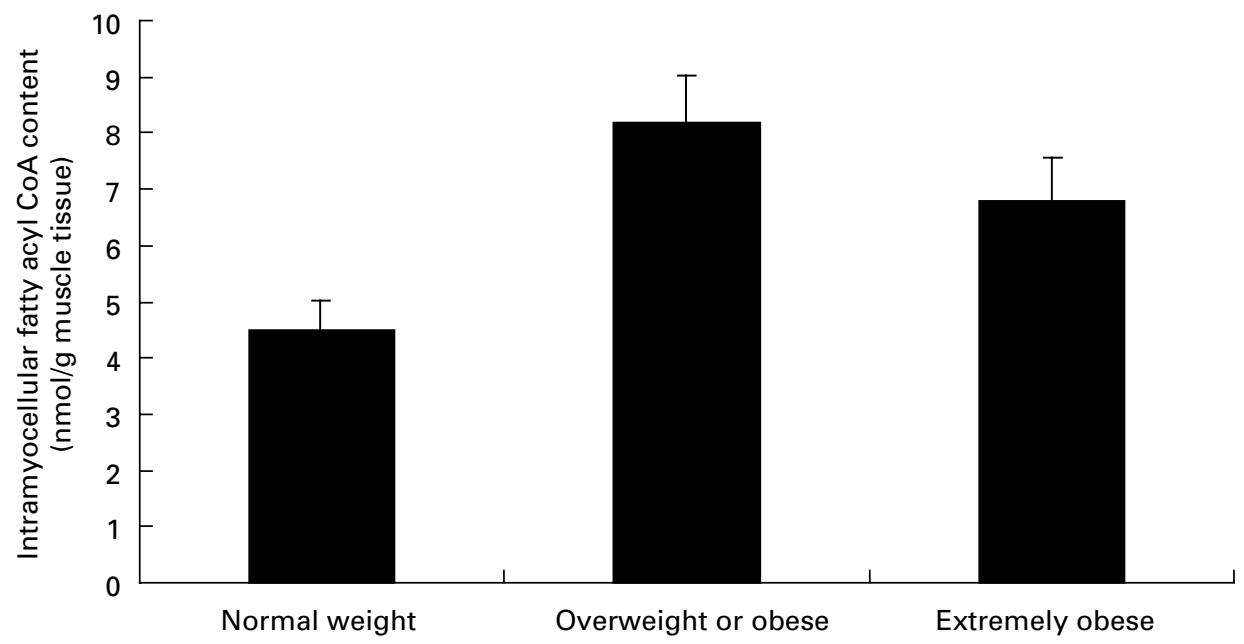

Fig. 2. Lipid oxidation and accumulation with obesity. (a) In vitro fatty acid oxidation in rectus abdominus muscle strips. (b) Intramyocellular fatty acyl-CoA content from rectus abdominus muscle strips. Values are means with their standard errors represented by vertical bars. (Redrawn from Hulver et al. 2003.)

in the indirect activation of PKC, as they are the precursors for DAG formation. DAG levels are elevated in many models of insulin resistance (Schmitz-Peiffer, 2002) and these intermediates directly activate PKC (Kishimoto et al. 1980; Nishizuka, 1984; Ishizuka et al. 1990; Kawakami et al. 2002).

\section{What is causing intramyocellular lipid accumulation?}

It is a logical assumption that fatty acyl-CoA accumulate in the cytosol of skeletal muscle as a result of decreased fatty acid oxidation and/or increased fatty acid uptake. This notion is supported by work from Kelley and colleagues (Kelley \& Simoneau, 1994; Kelley et al. 1999), who have demonstrated blunted fatty acid utilization and increased fatty acid uptake in skeletal muscle from patients with insulin-resistant disease states, such as obesity and type 2 diabetes. Previous work (Kim et al. 2000; Hulver et al. 2003) has shown decrements in skeletal muscle fatty acid oxidation in both whole homogenate and intact muscle preparations with obesity. Measurement of lipid metabolism in muscle strips from lean, moderately-obese and morbidly-obese patients (Hulver et al. 2003) has shown that fatty acid oxidation is reduced with morbid obesity but not with moderate obesity (Fig. 2(a)). In the same samples fatty acyl-CoA content is markedly higher in muscle from moderately- and morbidly-obese patients but not in muscle from lean patients (Fig. 2(b)). These findings are interesting, because fatty acyl-CoA are elevated in the moderately-obese tissue despite the presence of a high capacity to oxidize lipids. Furthermore, skeletal muscle from moderately- and morbidly-obese patients is equally insulin resistant (Fig. 1(a)). These data imply that a reduction in fatty acid oxidation is not a necessity for increased intramyocellular fatty acyl-CoA accumulation and suggest that mechanism(s) other than a blunted fatty 


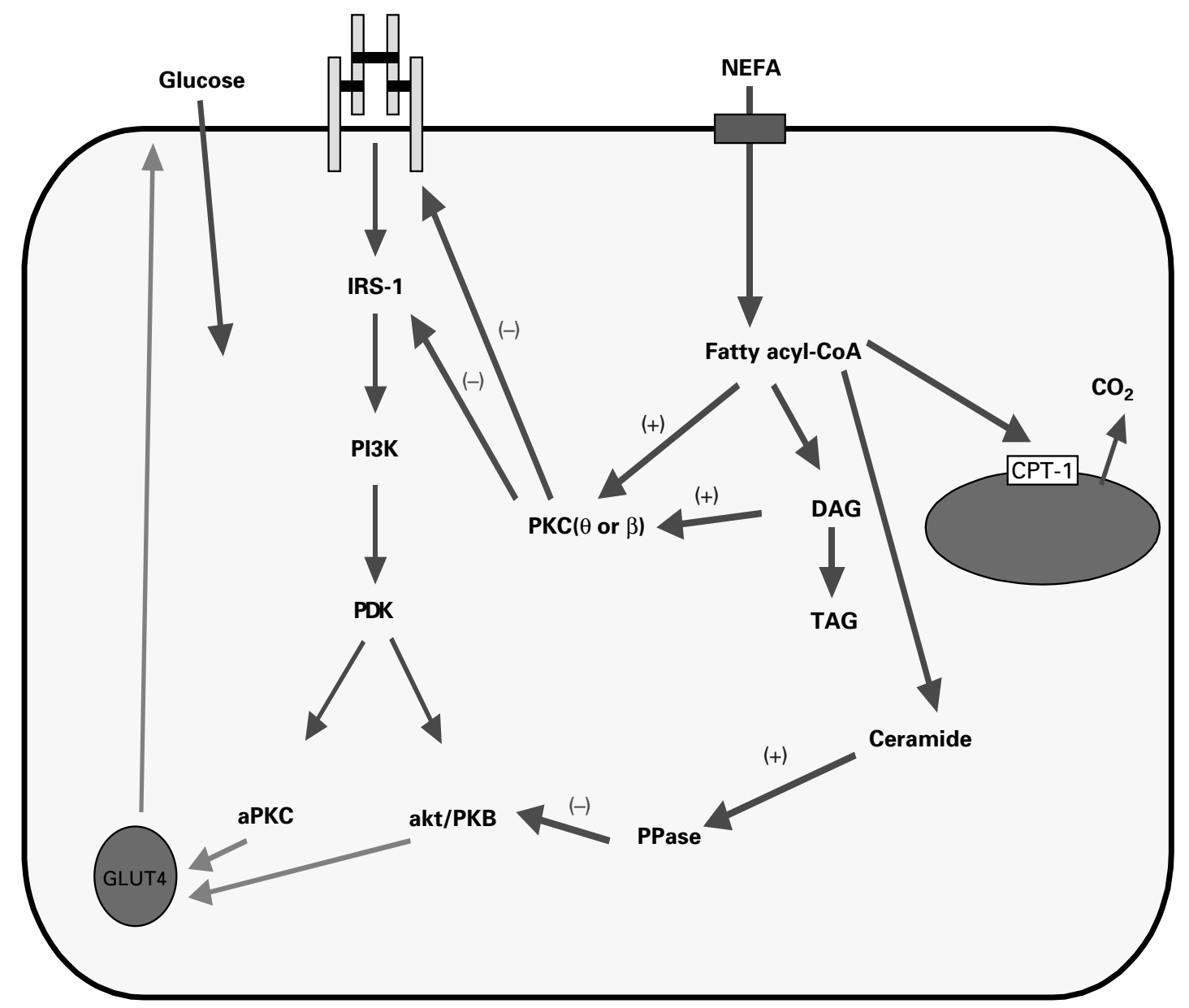

Fig. 3. Some of the cellular mechanisms that link intramyocellular lipid accumulation with insulin resistance. DAG, diacylglycerols; TAG, triacylglycerols; IRS-1, insulin receptor substrate 1; PI3K, phosphatidylinositol 3-kinase; PDK, phosphatidylinositol-dependent kinase; akt/PKB, protein kinase B; PKC, protein kinase C; aPKC, atypical protein kinase C; PPase, protein phosphatase; CPT-1, carnitine palmitoyltransferase 1; (+), activation; (-), inhibition.

acid oxidation may be responsible for intramyocellular fatty acyl-CoA accumulation.

Greater fatty acid uptake may be a potential mechanism responsible for the accumulation of intramyocellular lipids. Work from the authors' group has demonstrated that plasma lipids are elevated with obesity (MacLean et al. 2000). Thus, intramyocellular lipid accumulation may be a result of greater lipid supply to skeletal muscle and/or up regulated fatty acid transport into the muscle. To date, there have been no reports that have compared skeletal muscle fatty acid uptake in insulin-resistant and insulinsensitive muscle. However, the findings of a higher accumulation of fatty acyl-CoA in moderately-obese skeletal muscle, despite having the same levels of fatty acid oxidation compared with lean controls, suggest that fatty acid uptake is a likely culprit. Thus, it has been hypothesized that sarcolemmal and cytosolic transport of fatty acids is up regulated in insulin-resistant muscle, and experiments are currently underway to compare skeletal muscle fatty acid uptake in intact muscle strips from lean and obese patients.

\section{Conclusion}

Fig. 3 demonstrates the understanding of the link between intramyocellular lipids and insulin resistance. Increases in the intramyocellular concentrations of lipid intermediates (i.e. fatty acyl-CoA, ceramides and DAG) not only correlate with insulin resistance, but also directly and indirectly alter insulin signalling. Phosphorylation of serine and threonine residues on the insulin receptor and insulin receptor substrate 1 have been shown to be the steps in which insulin signalling is altered. PKC has been shown to be activated by various intramyocellular lipids and is believed to be the molecule responsible for the phosphorylation of the serine and threonine residues. The exact mechanism responsible for the increased accumulation of fatty acyl-CoA and DAG is unclear at this time. However, a reduction in fatty acid oxidation does not appear to be necessary for the accumulation of fatty acyl-CoA within skeletal muscle. Thus, an up-regulation of skeletal muscle fatty acid uptake may be responsible for increased skeletal muscle lipid accumulation. 


\section{References}

Azevedo JL Jr, Carey JO, Pories WJ, Morris PG \& Dohm GL (1995) Hypoxia stimulates glucose transport in insulin-resistant human skeletal muscle. Diabetes 44, 695-698.

Bachmann OP, Dahl DB, Brechtel K, Machann J, Haap M, Maier T, Loviscach M, Stumvoll M, Claussen CD, Schick F, Haring HU \& Jacob S (2001) Effects of intravenous and dietary lipid challenge on intramyocellular lipid content and the relation with insulin sensitivity in humans. Diabetes 50, 2579-2584.

Bossenmaier B, Mosthaf L, Mischak H, Ullrich A \& Haring HU (1997) Protein kinase C isoforms beta 1 and beta 2 inhibit the tyrosine kinase activity of the insulin receptor. Diabetologia 40, 863-866.

Bronfman M, Morales MN \& Orellana A (1988) Diacylglycerol activation of protein kinase $\mathrm{C}$ is modulated by long-chain acyl-CoA. Biochemical and Biophysical Research Communications 152, 987-992.

Caro JF, Sinha MK, Raju SM, Ittoop O, Pories WJ, Flickinger EG, Meelheim D \& Dohm GL (1987) Insulin receptor kinase in human skeletal muscle from obese subjects with and without noninsulin dependent diabetes. Journal of Clinical Investigation 79, 1330-1337.

Chavez JA, Knotts TA, Wang LP, Li G, Dobrowsky RT, Florant GL \& Summers SA (2003) A role for ceramide, but not diacylglycerol, in the antagonism of insulin signal transduction by saturated fatty acids. Journal of Biological Chemistry 278, 10297-10303.

Cooney GJ, Thompson AL, Furler SM, Ye J \& Kraegen EW (2002) Muscle long-chain acyl CoA esters and insulin resistance. Annals of the New York Academy of Sciences 967, 196-207.

Cortright RN, Azevedo JL Jr, Zhou Q, Sinha M, Pories WJ, Itani SI \& Dohm GL (2000) Protein kinase C modulates insulin action in human skeletal muscle. American Journal of Physiology 278, E553-E562.

Dohm GL, Tapscott EB, Pories WJ, Dabbs DJ, Flickinger EG, Meelheim D, Fushiki T, Atkinson SM, Elton CW \& Caro JF (1988) An in vitro human muscle preparation suitable for metabolic studies. Decreased insulin stimulation of glucose transport in muscle from morbidly obese and diabetic subjects. Journal of Clinical Investigation 82, 486-494.

Dolan PL, Tapscott EB, Dorton PJ \& Dohm GL (1993) Contractile activity restores insulin responsiveness in skeletal muscle of obese Zucker rats. Biochemical Journal 289, 423-426.

Ellis BA, Poynten A, Lowy AJ, Furler SM, Chisholm DJ, Kraegen EW \& Cooney GJ (2000) Long-chain acyl-CoA esters as indicators of lipid metabolism and insulin sensitivity in rat and human muscle. American Journal of Physiology 279, E554-E560.

Goodyear LJ, Giorgino F, Sherman LA, Carey J, Smith RJ \& Dohm GL (1995) Insulin receptor phosphorylation, insulin receptor substrate-1 phosphorylation, and phosphatidylinositol 3-kinase activity are decreased in intact skeletal muscle strips from obese subjects. Journal of Clinical Investigation 95, 2195-2204.

Griffin ME, Marcucci MJ, Cline GW, Bell K, Barucci N, Lee D, Goodyear LJ, Kraegen EW, White MF \& Shulman GI (1999) Free fatty acid-induced insulin resistance is associated with activation of protein kinase $\mathrm{C}$ theta and alterations in the insulin signaling cascade. Diabetes 48, 1270-1274.

Hulver MW, Berggren JR, Cortright RN, Dudek RW, Thompson RP, Pories WJ, MacDonald KG, Cline GW, Shulman GI,
Dohm GL \& Houmard JA (2003) Skeletal muscle lipid metabolism with obesity. American Journal of Physiology 284, E741-E747.

Ishizuka T, Cooper DR, Hernandez H, Buckley D, Standaert M \& Farese RV (1990) Effects of insulin on diacylglycerolprotein kinase $\mathrm{C}$ signaling in rat diaphragm and soleus muscles and relationship to glucose transport. Diabetes 39, 181-190.

Itani SI, Ruderman NB, Schmieder F \& Boden G (2002) Lipidinduced insulin resistance in human muscle is associated with changes in diacylglycerol, protein kinase C, and IkappaBalpha. Diabetes 51, 2005-2011.

Itani SI, Zhou Q, Pories WJ, MacDonald KG \& Dohm GL (2000) Involvement of protein kinase $\mathrm{C}$ in human skeletal muscle insulin resistance and obesity. Diabetes 49, 1353-1358.

Kawakami T, Kawakami Y \& Kitaura J (2002) Protein kinase C beta (PKC beta): normal functions and diseases. Journal of Biochemistry (Tokyo) 132, 677-682.

Kelley DE, Goodpaster BH, Wing RR \& Simoneau JA (1999) Skeletal muscle fatty acid metabolism in association with insulin resistance, obesity, and weight loss. American Journal of Physiology 277, E1130-E1141.

Kelley DE \& Simoneau JA (1994) Impaired free fatty acid utilization by skeletal muscle in non-insulin-dependent diabetes mellitus. Journal of Clinical Investigation 94, 2349-2356.

Kim JY, Hickner RC, Cortright RL, Dohm GL \& Houmard JA (2000) Lipid oxidation is reduced in obese human skeletal muscle. American Journal of Physiology 279, E1039-E1044.

Kishimoto A, Takai Y, Mori T, Kikkawa U \& Nishizuka Y (1980) Activation of calcium and phospholipid-dependent protein kinase by diacylglycerol, its possible relation to phosphatidylinositol turnover. Journal of Biological Chemistry 255, 2273-2276.

Long SD \& Pekala PH (1996) Lipid mediators of insulin resistance: ceramide signalling down-regulates GLUT4 gene transcription in 3T3-L1 adipocytes. Biochemical Journal 319, 179-184.

McGarry JD (2002) Dysregulation of fatty acid metabolism in the etiology of type 2 diabetes. Diabetes 51, 7-18.

MacLean PS, Bower JF, Vadlamudi S, Green T \& Barakat HA (2000) Lipoprotein subpopulation distributions in lean, obese, and type 2 diabetic women: a comparison of African and white Americans. Obesity Research 8, 62-70.

Nishizuka Y (1984) Turnover of inositol phospholipids and signal transduction. Science 225, 1365-1370.

Oakes ND, Bell KS, Furler SM, Camilleri S, Saha AK, Ruderman NB, Chisholm DJ \& Kraegen EW (1997) Diet-induced muscle insulin resistance in rats is ameliorated by acute dietary lipid withdrawal or a single bout of exercise: parallel relationship between insulin stimulation of glucose uptake and suppression of long-chain fatty acyl-CoA. Diabetes 46, 2022-2028.

Schmitz-Peiffer C (2002) Protein kinase C and lipid-induced insulin resistance in skeletal muscle. Annals of the New York Academy of Sciences 967, 146-157.

Shoyab M (1985) Long-chain fatty acyl-coenzyme As activate both the ligand-binding and protein kinase activities of phorboid and ingenoid receptor. Archives of Biochemistry and Biophysics 236, 435-440.

Shulman GI (2000) Cellular mechanisms of insulin resistance. Journal of Clinical Investigation 106, 171-176.

Zhou Q, Dolan PL \& Dohm GL (1999) Dephosphorylation increases insulin-stimulated receptor kinase activity in skeletal muscle of obese Zucker rats. Molecular and Cellular Biochemistry 194, 209-216. 